\title{
Article
}

\section{Reasoning deficits among illicit drug users are associated with aspects of cannabis use}

Fisk, John, Morley, Andy M, Hadjiefthyvoulou, Florentia and Montgomery, Catharine

Available at http://clok.uclan.ac.uk/23676/

Fisk, John ORCID: 0000-0002-2981-0870, Morley, Andy M ORCID: 0000-00031942-1983, Hadjiefthyvoulou, Florentia and Montgomery, Catharine (2014) Reasoning deficits among illicit drug users are associated with aspects of cannabis use. Cognitive Processing, 15 (4). pp. 523-534. ISSN 1612-4782

It is advisable to refer to the publisher's version if you intend to cite from the work. http://dx.doi.org/10.1007/s10339-014-0616-2

For more information about UCLan's research in this area go to http://www.uclan.ac.uk/researchgroups/ and search for <name of research Group>.

For information about Research generally at UCLan please go to http://www.uclan.ac.uk/research/

All outputs in CLoK are protected by Intellectual Property Rights law, including Copyright law. Copyright, IPR and Moral Rights for the works on this site are retained by the individual authors and/or other copyright owners. Terms and conditions for use of this material are defined in the policies page.

\section{CLoK}

Central Lancashire online Knowledge www.clok.uclan.ac.uk

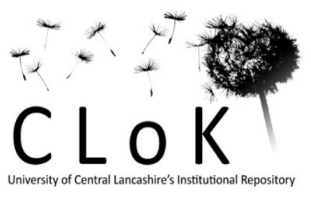


Reasoning deficits among illicit drug users are associated with aspects of cannabis use.

John E. Fisk ${ }^{\mathrm{a}}$; Andy M. Morley ${ }^{\mathrm{a}}$; Florentia Hadjiefthyvoulou ${ }^{\mathrm{b}}$,

Catharine Montgomery ${ }^{\mathrm{c}}$

a: School of Psychology, University of Central Lancashire, Preston PR1 2HE, UK

b: Division of Psychology, Nottingham Trent University, Nottingham NG1 4BU, UK

c: School of Natural Sciences \& Psychology, Liverpool John Moores University, Liverpool, L3 3AF, UK

Running Head: Reasoning deficits among substance users

\section{Corresponding author:}

Professor John E Fisk, PhD

School of Psychology

University of Central Lancashire

Preston PR1 2HE

United Kingdom

Tel 44 (0) 1772894465

Fax 44 (0) 1772892925

e-mail: jfisk@uclan.ac.uk 


\begin{abstract}
Background. Deficits in deductive reasoning have been observed among ecstasy/polydrug users. The present study seeks to investigate dose-related effects of specific drugs and whether these vary with the cognitive demands of the task.

Methods. One hundred and five participants (mean age 21.33, S.D. 3.14; 77 females, 28 males) attempted to generate solutions for eight one-model syllogisms and one syllogism for which there was no valid conclusion (NVC). All of the one model syllogisms generated at least one valid conclusion and six generated two valid conclusions. In these six cases one of the conclusions was classified as common and the other as non-common.

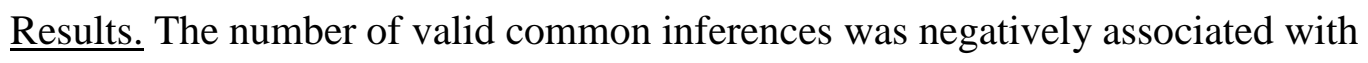
aspects of short term cannabis use and with measures of IQ. The outcomes observed were more than simple post intoxication effects since cannabis use in the 10 days immediately before testing was unrelated to reasoning performance. Following adjustment for multiple comparisons, the number of non-common valid inferences was not significantly associated with any of the drug use measures.

Conclusions. Recent cannabis use appears to impair the processes associated with generating valid common inferences while not affecting the production of non common inferences. It is possible, therefore, that the two types of inference may recruit different executive resources which may differ in their susceptibility to cannabis-related effects.
\end{abstract}

Key words: syllogism, inference, ecstasy drug, cannabis, cocaine 


\section{INTRODUCTION}

The purpose of the present paper is to evaluate the relative integrity of reasoning processes in users of legal and illicit drugs. In order to achieve this we utilise a well tested experimental paradigm which has assumed a fundamental role in the study of deductive reasoning: the syllogism. A syllogism contains two premises from which the participant attempts to derive logically necessary conclusions. Syllogisms can be framed in abstract form or in everyday language as in the following example:

1. Some students are in employment

2. All those who are in employment are paid money

Therefore it can be concluded that:

Some students are paid money

Some of those who are paid money are students

Or in abstract form:

1. Some A are B

2. All B are $\mathrm{C}$

Therefore

Some A are C

Some $\mathrm{C}$ are A.

Syllogisms are known to vary in terms of difficulty. Some pairs of premises can be readily solved allowing valid conclusions to be drawn by most people. In other cases, only a small percentage of those who attempt to derive a conclusion are successful (Johnson-Laird, 1983). The major theoretical perspective which has emerged in the context of syllogistic reasoning and in deductive reasoning in general is mental models theory. It has been asserted that individuals construct a model or models of the premises through which they derive potential conclusions. While some pairs of premises can be exhaustively represented in just a 
single model, the majority require the construction of two, or more often three, models in order to explore alternative formulations of the premises (Johnson-Laird, 1983). In turn, the construction of additional models places increased demands on working memory resources (Gilinsky \& Judd, 1994).

Indeed, the direct involvement of working memory in supporting syllogistic reasoning is demonstrated in studies utilising the dual task methodology. For example, utilising Baddeley's working memory framework, administration of a random generation task, known to load on the central executive component of working memory, reduced syllogistic reasoning performance while other concurrent tasks loading on the phonological loop and visuo-spatial sketchpad did not (Gilhooley et al. 1993). In a later study (Gilhooley et al. 1999), the performance of those participants who were more proficient in solving syllogistic reasoning problems were similarly affected by dual tasks loading on the central executive while those who were less proficient appeared to be using a less cognitively demanding strategy which was less reliant on central executive resources. After training in deductive reasoning was administered, this latter group appeared to change their approach adopting a strategy which was more dependent on working memory resources and, accordingly, more susceptible to dual tasks loading on the central executive.

It has long been argued that the processes through which mental models are constructed and evaluated, so as to derive valid conclusions for syllogisms, are the same as those which underpin real world reasoning in more everyday contexts (Bucciarelli \& Johnson-Laird, 1999; Galotti, 1989; Morris \& Nisbett, 1993). While it is frequently the case that everyday decisions may be intuitive and heuristic in nature, there are occasions when judgements require a more rational analysis and in these situations mental models theory provides a widely accepted account of how this is achieved (Verschueren, Schaeken, \& d'Ydewalle, 2005). Indeed Politzer (2011) has shown that, in everyday reasoning, the 
evaluation of natural syllogisms, i.e., those that reflect real world categorical relationships, appears to follow a process which is indistinguishable from strategies commonly used in laboratory based syllogistic reasoning tasks like those presented here. Thus, the study of syllogistic reasoning provides an insight into the efficacy of reasoning processes in more everyday contexts.

It is clear from previous research that ecstasy/polydrug users are impaired on tests of syllogistic reasoning (Fisk et al. 2005; Montgomery et al. 2005). Furthermore, while Montgomery et al. (2005) linked this outcome specifically with ecstasy use, Fisk et al. (2005) found that various measures of ecstasy, cannabis and cocaine were associated with errors on the task. However, dose-related effects have yet to be systematically examined and the possibly confounding effects of legal drugs such as alcohol and tobacco have been overlooked. It is important to establish that unambiguous dose-related effects exist. In previous research (Fisk et al. 2005; Montgomery et al. 2005) where dose related effects were apparently obtained, nonusers of specific drugs (with use coded as zero) were included in the sample. The problem with this is that the significant correlation and regression coefficients that were obtained might have been due to the absence of use within the drug naïve participants (i.e., the group effect) rather than a trend within the drug using participants. This being the case, the possibility that some premorbid or lifestyle difference between the groups unrelated to drug use may have been responsible for the associations that were observed cannot be excluded. In the evaluation of dose-related effects here, we will limit the analysis to those individuals actually using each specific drug. This will also allow for the increasing prevalence of polysubstance use to be addressed more effectively. For example, while less than half of Fisk et al's (2005) ecstasy users were also using cocaine, the increased use of this drug among young adult recreational users has meant that it is not unusual to find that up to 
70 or $80 \%$ of current users of ecstasy are also cocaine users while virtually all are using cannabis (e.g., Hadjiefthyvoulou et al. 2011).

In a further departure from Fisk et al's (2005) approach, while in the present study we use the same four one model syllogisms as they and Montgomery et al. (2005) used, here we have not included the more difficult two and three model problems. Fisk et al. (2005) and Montgomery et al. (2005) found that these elicited a success rate that was little better than chance among their participants. So instead, we have included an additional four one model problems. Furthermore, while Fisk et al. (2005) and Montgomery et al. (2005) treated correct responses to one model problems in an undifferentiated manner, in the present paper we will seek to classify responses in a different way. Unlike two and three model problems which generate only a single valid inference, most one model problems generate two valid conclusions. However, Johnson-Laird (1983) has demonstrated that only a minority of participants manage to produce both conclusions for one model syllogisms and although equally valid, some conclusions are produced far more often than others (Khemlani \& Johnson-Laird, 2012). Using the example above, individuals are more likely to produce the conclusion: 'Some students are paid money' but less likely to generate the second valid conclusion: 'Some of those who are paid money are students'. This tendency is believed to be at least partially due to the ordering of terms in the premises (known as the figural effect), so, for example, in the syllogism set out above, conclusions of the form A to $\mathrm{C}$ are more likely to be produced than $\mathrm{C}$ to $\mathrm{A}$. Furthermore, when at least one premise contains the particular quantifier ('Some') then the conclusion is also more likely to contain it (an example of the atmosphere effect) (Johnson-Laird, 1983). Thus while not necessarily placing additional demands on working memory, the ability to produce the less common conclusion may depend on other aspects of executive functioning, for example, the ability to inhibit prepotent responses or to switch cognitive set away from the common response so as to consider other 
possibilities. Thus it is predicted that illicit drug use will be negatively associated with the number of valid inferences produced and that this tendency will be particularly evident in relation to the less common inferences, where the cognitive demands of the task are potentially greater.

\section{METHOD.}

$\underline{\text { Participants. }}$

One hundred and five participants (mean age 21.33, S.D. 3.14; 77 females, 28 males) were recruited opportunistically through direct approach to undergraduate students at Liverpool John Moores University and the University of Central Lancashire in the United Kingdom. Potential participants were recruited through the use of posters displayed around campus and thereafter through word-of mouth. While prospective participants were informed that the focus of the study was concerned with illicit drugs, those who indicated that their use was largely restricted to legal drugs (alcohol and tobacco) were also included. In the event most participants were polydrug users and the prevalence of multiple drug use may be seen in Table 1.Those participating in the study were asked to abstain from taking cannabis for at least 24 hours and other illicit drugs for seven days prior to testing. Median periods of abstinence were three, six, and four weeks for cannabis, ecstasy, and cocaine respectively. <<Insert Table 1 about here.>>

\section{Materials.}

The use of legal and illegal drugs was assessed by means of a self report questionnaire. For cannabis, cocaine and ecstasy, participants indicated their current frequency of use, their level of recent use, and the period of abstinence. They also indicated which other illicit drugs they had previously used. Estimates of alcohol and tobacco use were also obtained including current consumption levels, recent use, and duration of use. 
Demographic variables, including age and gender, were recorded. Fluid intelligence was also assessed through Raven's progressive matrices (Raven et al. 1998). In order to provide information on longer term consumption patterns, for the most commonly used drugs (ecstasy, cannabis and cocaine), for each year since they commenced use, participants estimated the typical dose that they ingested in a representative session. They also estimated their typical frequency of use (number of sessions per week) during that year. These data were subsequently used to produce estimates of annual average long term dose and long term average frequency of use, as well as total lifetime use. Regrettably, the responses of 31 participants relating to these longer term consumption patterns were lost during the relocation of one of our laboratories. Thus of the whole sample, 74 out of a total of 105 completed the full version of the self-report drug use questionnaire.

Syllogistic reasoning: All syllogisms were taken from Johnson-Laird (1983, pp102103) where for each legitimate pair of premises the logically valid inferences (if any) are indicated. Participants attempted to generate solutions for eight one-model syllogisms and one syllogism for which there was no valid conclusion (NVC). The one model syllogisms were the same as those used in much of the previous research literature (see the review by Khemlani \& Johnson-Laird, 2012). All of the one model syllogisms generated at least one valid conclusion and six generated two valid conclusions. In these six cases one of the conclusions was classified as common and the other as non-common. The classification was based on data published by Johnson-Laird (1983). For the common variant, between 40 and 95\% (average 61\%) of Johnson-Laird's participants generated the correct response while for the non common alternative, the equivalent figures were between 0 and $25 \%$ (average 14\%). The syllogisms used in the study were presented in random order. Participants were introduced to the concept of a syllogism, and examples (concrete and abstract) were provided. Examples and explanations were also provided for some correct and incorrect 
inferences, and Venn diagrams were used for purposes of illustration. Participants were told to generate as many conclusions as possible for each pair of premises. They were told that no pair generated more than two valid conclusions, some only generated one, and some had no valid conclusions. In addition, they were provided with a list of the eight possible solutions that can be generated over all the pairs of premises. The syllogisms were presented in a booklet, in abstract form as in the example set out above. In each case, the two premises were printed, followed by the instruction to 'Please write down all valid conclusions'. Two boxes were provided underneath for the participant to record their responses. A response was deemed correct if it followed necessarily from the premises or in the case of the NVC syllogism, if the participant indicated that no valid conclusions were possible. With regard to the common conclusions, with eight valid one-model syllogisms, the maximum number of correct (logical) common inferences was eight. Six of these one model syllogisms also generated a second but, as demonstrated by Johnson-Laird (1983), less common valid inference. Thus, the maximum number of correct (logical) non common inferences was six. Procedure.

Participants provided verbal informed consent. The tests were administered in the following order: background drug use questionnaire, Ravens progressive matrices and the syllogistic reasoning test. A range of other measures was also administered the results of which are outside the scope of the present study. At the end of the session, participants were debriefed, paid 20 UK pounds in the form of a supermarket (grocery store) gift card, and provided with drug education leaflets. The study was approved by the Ethics Committees of Liverpool John Moores University, and the University of Central Lancashire and was administered in accordance with the ethical guidelines of the British Psychological Society. All aspects of the Helsinki Declaration were observed except that verbal consent was obtained so as to protect the anonymity of the illicit drug users within our sample. 


\section{Design.}

Associations between the number of valid syllogistic inferences and respectively background variables, alcohol and tobacco use and various indicators of legal and illegal drug use and were explored. While some means of controlling the Type 1 error rate is required it is now well established that full Bonferroni correction greatly inflates the likelihood of Type 2 error (Nakagawa, 2004). Where test results are conditionally dependent, (as is the case with the present study, where there are two interrelated outcome variables and multiple intercorrelated drug use measures) full Bonferroni correction is known to be inappropriate (Bland \& Altman, 1995; Narum, 2006; Pike, 2010). Thus, an alternative to full Bonferroni correction has been adopted here, which focusses on controlling the False Discovery Rate (FDR), a technique which is well suited to situations where the reported outcomes are not independent (Benjamini \& Yekutieli, 2001). This involves controlling the proportion of occasions where true null hypotheses are falsely rejected giving rise to 'false discoveries'. Computational methods are available for calculating the critical value for alpha (also known as the q value) which controls the FDR at a given level (e.g., Pike, 2010). The FDR rate in the present study was set to .05 which implies that the proportion of significant outcomes which are actually false discoveries is limited no more than $5 \%$.There is a related procedure for calculating the critical alpha value which limits the Family Wise Error rate (FWE) without greatly inflating the risk of a Type 2 error, as is the case with full Bonferroni correction (Benjamini \& Yekutieli, 2001; Narum, 2006). It is this critical level and the related FDR which has been used to identify those outcomes in Table 2 which can be regarded as statistically significant with the FWE $<.05$ and FDR $<.05$, two tailed.

\section{RESULTS}




\section{Background Data}

Examination of Table 1 reveals the extent of polydrug use among the sample. Almost all participants used alcohol and 30\% used tobacco. The most commonly used illicit drug was cannabis with $63 \%$ of the sample using this drug. The proportions using ecstasy and cocaine were similar at approximately 50\%. Less commonly used were amphetamine (19\%), ketamine (24\%) and LSD (17\%) and in these three cases only 7, 9 and 4 individuals reported using respectively amphetamine, ketamine, and LSD during the previous 3 months and in all but 4 cases these individuals described their use as 'occasional' (as opposed to 'frequently' and 'always').

For the major illicit drugs, inspection of Table 2 reveals that cannabis users were using approximately weekly and on average had consumed almost 12 joints during the previous 30 days. Those using cocaine took it approximately bi-weekly and on average had consumed 8 lines during the previous 30 days. Ecstasy was consumed roughly once a month and the individuals concerned had taken on average 4-5 tablets during the previous 30 days. Table 2 also contains indicators of long term use with total lifetime consumption averaging 2427 joints, 717 lines, and 675 tablets for cannabis, cocaine, and ecstasy respectively. $<<$ Insert Table 2 about here $>>$

\section{Syllogistic Reasoning Performance.}

Consistent with Johnson-Laird's (1983) reported data, participants were better at generating common responses compared to the non-common variants. In the present study on average participants generated $56 \%$ of the valid common conclusions while averaged over all relevant one model problems $61 \%$ of Johnson-Laird's participants produced the valid common conclusions. The equivalent figures for the non-common variant was $33 \%$ in the present study compared with just $15 \%$ among Johnson-Laird's participants. Thus the 
participants in the present study performed similarly to Johnson-Laird's in terms of the common conclusions but did considerably better in relation to the non-common conclusions.

\section{Associations between Background Variables, Indicators of Drug use and Number of}

\section{Correct Inferences.}

Perhaps unsurprisingly, fluid intelligence was positively associated with reasoning performance although the magnitude of the association was higher for common inferences. None of the alcohol use measures were significantly associated with reasoning performance. Without adjustment for multiple comparisons, tobacco (cigarettes per day) was negatively associated with both common and non-common inferences, $\mathrm{p}<.05$, two tailed, in both cases. Furthermore, of the illicit drugs, four of the cannabis use measures were negatively associated with reasoning performance at $p<.05$ or less, two tailed (in all cases these relate to valid common inferences); the long term average dose of ecstasy was also negatively related to the number of valid common inferences, $\mathrm{p}<.05$. Lastly, the current frequency of cocaine use was negatively associated with non-common inferences. Analysis revealed that the largest of these probabilities, i.e., $\mathrm{p}=.033$, was associated with an estimated FDR=.152 implying that up to $15.2 \%$ of these apparently significant outcomes were false discoveries.

Further analysis (Pike, 2010) demonstrated that an alpha value less than or equal to .002 controlled the FDR $<.05$. On this basis only two of the associations reported in Table 2 were statistically significant, i.e., fluid IQ was positively, and consumption of cannabis during the previous 30 days negatively, associated with the number of valid common inferences. From an alternative perspective, a critical value of .01132 controls the FWE $<.05$ two tailed (Narum, 2006). On this criterion, two additional statistically significant associations emerged: the current frequency of cannabis use was negatively associated with the number of valid common inferences and the fluid IQ measure was positively associated 
with the number of non-common valid inferences. While Benjamini and Yekutieli's (2001) procedure provides a means of selecting an alpha value which, relative to more conventional approaches, is less likely to inflate the type 2 error rate, the power of the statistical procedure to detect a significant association remains dependent on the sample size. It is clear from Table 2 that while the measures of short term use (e.g., the previous 30 days) for the most commonly used drug, cannabis, was based on a sample size of 62 , longer term cannabis trends, such as average dose per session, was based on a sample of 46 . The corresponding figures for short and longer term cocaine use were 38 and 26 respectively. The reduced number of cocaine users is clearly associated with a reduction in statistical power. For example, with short term consumption data for 38 cocaine users, the power to detect a correlation of -.389 (the outcome in relation to cannabis) with alpha $=.002$ (FDR adjusted) would be .338 , one-sided. With regard to the long term cocaine data, with a sample size of 26 , the power to detect a correlation of -.324 (again, the outcome in relation to cannabis) with the same FDR adjusted alpha value would be just .101, one-sided. These low levels of power should be borne in mind when considering the apparently non significant outcomes in Table 2.

Returning to the significant associations that emerged in Table 2, two of these relate to the recent use of cannabis: current frequency of use and amount consumed during the previous 30 days. It is possible that these associations may reflect post intoxication effects connected with very recent use of the drug. To explore this possibility, we examined cannabis use during the ten days prior to testing and it emerged that 22 participants had used the drug within this period. Mean consumption among this group was 3.32 joints (SD 3.34). However, consumption was unrelated to reasoning performance, $r=.026(p=.910)$ and $-.089(p=.693)$ for common and non-common inferences respectively. 
As noted above, IQ was significantly associated with reasoning performance. It is possible that those with higher short term levels of cannabis use might score lower on the IQ measure, leaving open to question whether the cannabis-related deficits observed were in fact attributable to IQ or indeed whether cannabis use adversely affects both reasoning and IQ. In order to examine this question, the partial correlations between cannabis use and common valid inferences were calculated controlling for differences in IQ. The partial correlation between cannabis use during the previous 30 days and valid common inferences remained statistically significant, $r=-.346, \mathrm{p}=.007, \mathrm{FWE}<.05$; however, the correlation between the current frequency of cannabis use and common inferences was reduced to below statistical significance, $r=-.254, p=.050$. Thus it appears that IQ and cannabis-related effects are, at least to a degree, independent.

In order to establish whether or not the above mentioned short term cannabis-related effects are independent of the effects of cocaine and ecstasy two regressions were run. In both cases the dependent variable was the number of valid common syllogistic conclusions. In the first independent variables were, respectively, consumptions of cannabis, cocaine and ecstasy during the 30 days prior to testing. In the second independent variables were, respectively, the current frequencies of cannabis, cocaine and ecstasy use. For these two regressions, values for nonusers of specific drugs were coded as zero. In addition both regressions contained zero-one variables respectively for cannabis, cocaine and ecstasy in order to distinguish nonusers of each drug from users. This was done in order to remove the effects of simple group related differences that may be unrelated to direct dose-related effects. In the first regression the independent variables accounted for statistically significant variance in the number of correct common inferences, $\mathrm{R}^{2}=.156, \mathrm{~F}(6,94)=2.90, \mathrm{p}=.012$. Only the consumption of cannabis during the 30 days prior to testing was statistically significant as a predictor, beta $=-.329, \mathrm{t}=-3.34, \mathrm{p}=.001$, semi partial correlation coefficient $=-.316$. The 
relationships between the consumption of the major illicit drugs, cannabis, cocaine, and ecstasy, during the 30 days prior to testing and the number of correct common syllogistic responses are displayed in Figures 1, 2, and 3. In the second regression the independent variables again accounted for statistically significant variance, $\mathrm{R}^{2}=.148, \mathrm{~F}(6,82)=2.37$, $\mathrm{p}=.037$. The current frequency of cannabis use was statistically significant as a predictor, beta $=-.275, \mathrm{t}=--2.53, \mathrm{p}=.013$, semi partial correlation coefficient $=-.258$. Interestingly the current frequency of cocaine use was also statistically significant, beta=-.275, $\mathrm{t}=--2.14$, $\mathrm{p}=.036$, semi partial correlation coefficient $=-.218$. The remaining predictors were not significant. The relationships between the current frequency of cannabis, cocaine and ecstasy use and the number of correct common syllogistic responses are displayed in Figures 4, 5, and 6.

\section{DISCUSSION}

On the basis of the results reported by Fisk et al. (2005) and Montgomery et al. (2005) it might have been expected that ecstasy would show an inverse dose-related effect with reasoning performance. However, only the long term average dose of ecstasy was significantly associated with reasoning performance on an unadjusted basis, and this was no longer significant at alpha values which controlled the FDR and FWE. Among the major illicit drugs, cannabis use emerged as the only robust correlate of reasoning performance and this only in relation to the number valid common inferences. At alpha values controlling the FDR $<.05$ or the FEW $<.05$, the current frequency of cannabis use and the number of joints consumed during the 30 days prior to testing were negatively associated with the number of valid common inferences. Thus it appears that recent aspects of cannabis use affect reasoning performance although the effects observed appear unlikely to be simple post intoxication effects. Interestingly the regression analyses revealed that the short term effects of cannabis 
on the number of correct common syllogistic inferences remaining statistically significant after controlling for the short term effects of cocaine and ecstasy. Although, on an unadjusted basis, the long term average dose and long term average frequency of cannabis use were correlated with the number of valid common inferences, these were no longer significant following controls for the FDR and FWE. Furthermore it is likely that these longer term cannabis use measures shared variance with the short term measures, raising the possibility that it is the latter that is behind the significant association with reasoning performance.

We are not alone in linking other illicit drugs with less effective reasoning processes. For example, a group of cocaine dependent individuals, who also had a history of recent cannabis use, did significantly worse on the similarities reasoning test (Cunha, Nicastri, de Andrade \& Bolla, 2010). Furthermore, in a group of polysubstance abusers who had requested treatment for drug related problems, measures of cocaine and cannabis use were selectively associated with deficits in analogical reasoning, in a dose related manner (Fernández-Serrano, Pérez-García, Río-Valle \& Verdejo-García, 2010). Therefore the presence of illicit drug-related reasoning deficits in the present sample of illicit drug users is not without precedent and while previous studies using syllogisms have linked this to ecstasy use (Fisk et al. 2005; Montgomery et al. 2005), the present results suggest that cannabis is also implicated. It is also worthy of note that Cunha et al's (2010) and Fernández-Serrano et al's (2010) results relate to drug dependent clinical groups while our focus here was recreational users. Furthermore, these studies (and others) have used reasoning paradigms with a substantial verbal component, raising the possibility that the deficits observed might reflect aspects of verbal processing rather than pure reasoning processes (Rodriguez-Moreno \& Hirsch, 2009). By way of contrast, our research materials were more abstract in nature and utilised only elementary verbal concepts. 
With regard to the underlying causes of cannabis-related effects, there is evidence to suggest that a history of cannabis use is associated with neural changes in areas which play a role in reasoning performance. For example, Kanayama et al (2004) found that relative to controls, cannabis users demonstrated higher activation levels in the PFC and anterior cingulate during the completion of a spatial working memory task and also showed activations in areas not normally seen in controls including the basal ganglia. Also using fMRI, Jager et al. (2006) found that while frequent (but moderate) cannabis users performed similarly to nonusers in tests of working memory and visuo-auditory selective attention, differences were observed in brain activity between users and nonusers in the superior parietal cortex during performance of the tasks. In a subsequent study Jager et al (2007) asked their participants to perform an associative learning task while undergoing fMRI. Lower activation levels among frequent cannabis users were observed in the medial temporal structures (especially the para-hippocampal area) and the right DLPFC, although paradoxically task performance was again unaffected. Kanayama et al. (2004) and Jager et al. $(2006 ; 2007)$ have suggested that the differential pattern of neural activity evident in cannabis users may reflect the need to recruit additional cognitive resources in order to maintain performance at a comparable level to nonusers.

Over a number of years, evidence has emerged that syllogistic reasoning is dependent on prefrontal cortical resources. For example, in two recent neuroimaging studies, Reverberi and co workers found that relative to simply recalling the premises, actively encoding them was associated with increased activity in left hemisphere prefrontal structures including Brodmann areas (BA) 44 and 45. During integration of the premises BA44/45 remained differentially activated along with BA6 and areas within the VLPFC (BA47), the parietal cortex (BA7) and the basal ganglia (Reverberi et al. 2010; 2012). Rodriguez-Moreno and Hirsch (2009) have also emphasised the role of more DLPFC structures (including left 
hemisphere BA 6, 8, 9, and 10), VLPFC structures, (e.g., BA47) and left hemisphere parietal regions (BA 7, 39, and 40) in syllogistic reasoning performance. Similar cortical regions were identified by Goel et al. (2000) in an early study investigating the cortical basis of reasoning with abstract syllogisms as are used in the present study. Thus the neural areas identified by Jager et al (2006; 2007) and Kanayama et al (2004) that were differentially activated in cannabis users also appear to play a prominent role in reasoning performance. For example, it is worthy of note that among the regions identified by Reverberi et al. $(2010 ; 2012)$ that were differentially recruited during the integration of the syllogistic premises i.e., the superior parietal cortex and basal ganglia, are the same as those that were identified by Jager et al (2006; 2007) and Kanayama et al (2004) as being potentially compromised among cannabis users during the performance of tasks loading on working memory. While these were not associated with performance deficits in working memory, it is possible that the greater cognitive demands associated with the reasoning task did result in the apparent cannabisrelated deficit. For example, it is worthy of note that among the regions identified by Reverberi et al. $(2010 ; 2012)$ that were differentially recruited during the integration of the syllogistic premises, i.e., the superior parietal cortex and basal ganglia, are the same as those that were identified by Jager et al $(2006 ; 2007)$ and Kanayama et al (2004) as being potentially compromised among cannabis users during the performance of tasks loading on working memory. While these were not associated with performance deficits in working memory it is possible that the greater cognitive demands associated with the reasoning task did result in the apparent cannabis-related deficit.

An additional unanticipated finding, in the present study, was the negative association between the number of (tobacco) cigarettes typically consumed daily and reasoning performance. Although statistically significant on an unadjusted basis these associations were not significant at alpha levels which controlled the FDR and FWE. Nonetheless the results 
remain noteworthy since the absolute magnitudes of the correlations were relatively high, based on generally small numbers of current smokers. Interestingly, Jacobsen et al (2007) note that most cannabis users also smoke tobacco and that the joint effects of these two psychoactive substances remains unclear. Indeed Jacobsen et al. (2007) found that verbal memory and learning was impaired among adolescent cannabis users (but not nonusers) during a period of nicotine withdrawal. Through fMRI it was established that the impairment was associated with disrupted frontoparietal connectivity. Thus it is possible that nicotine and cannabis may interact in terms of their effects potentially giving rise to reasoning impairments (Jacobsen et al 2007).

As expected, IQ was significantly associated with both the number of common and non-common inferences. As Khemlani and Johnson-Laird (2012) note since many intelligence test actually contain syllogistic or functionally equivalent problems a significant association might therefore be expected. However, perhaps surprisingly, there is relatively little current research into the association between the two constructs. In one of the few recent studies, Shikishima et al. (2009) found that syllogistic reasoning problems (including abstract, graphical and congruent) loaded highly on the general IQ construct ' $\mathrm{g}$ ' along with more conventional verbal and spatial tests of IQ. Indeed the respective loadings on $g$ of the three classes of measure were highest for the syllogistic reasoning measures. Furthermore genetic and environmental influences were consistent across the three separate performance classes, yielding a significant genetic correlation and a high degree of heritability for the latent ' $\mathrm{g}$ ' construct. In a subsequent study (Shikishima et al. 2011), syllogistic reasoning ability was observed to have similar characteristics as other indicators of intelligence, exhibiting a similar developmental trajectory, as well as exhibiting the usual relationship with socio-economic status. Furthermore, syllogistic reasoning was genetically correlated with aspects of scholastic achievement. Thus the significant relationship observed here between IQ 
and reasoning is consistent with current research findings in this area. The only surprising outcome was that the correlation between IQ and non common inferences was less that that with common inferences. Given that non common inferences are more demanding on cognitive resources, a stronger relationship with IQ might have been expected.

It is also surprising that, following controls for the FDR and FEW, none of the drug use measures were significantly associated with the number of non common valid inferences. At face value, this might be taken as evidence that that illicit drug use does not compromise whatever cognitive abilities and resources support the generation of non common inferences. It has been demonstrated that producing valid inferences recruits working memory (updating) resources (e.g., Gilhooly et al. 1993; 1999). It may be the case that other executive processes such as inhibition and shifting are required to switch the focus away from the dominant (common) response and search for other valid inferences. Research suggests that while ecstasy/polydrug users are impaired in updating component executive processes, shifting and inhibition are relatively spared (Murphy et al. 2009). Thus the present results may be a reflection of this.

Some limitations need to be acknowledged in relation to the present study. For example, we have used zero order and partial correlation. While regression analysis might have been preferable, the sample size was insufficient since it is effectively constrained by the smallest subset of illicit drug uses, i.e., cocaine users in the present case. While we might have coded non use of specific drugs as ' 0 ' the problem with this is that it potentially introduces group level effects which may be due to factors (e.g., premorbid or lifestyle differences) other than the particular drug in question. An additional factor mitigating against the use of regression, was the degree of intercorrelation between some of the drug use measures which would give rise to multicolinearity. 
Caution needs to be exercised when drawing inferences concerning the association observed here between cannabis and reasoning performance. While the existence of a doserelated relationship between a particular drug and cognitive performance is consistent with the possibility that increasing amounts of the drug have a tendency to directly affect performance, it remains possible that heavy users may exhibit premorbid or life style differences in relation to light users which may be, at least in part, responsible for the performance differences that we have observed. For example, there may be differences in diet, or sleep quality which might mediate the effects observed. Alternatively the higher doses consumed might reflect a tendency for increased impulsivity which might also have a direct effect on performance. The cannabis users in the present study were predominantly polydrug users and therefore it is possible that the effects observed may in fact reflect some sort of cocktail effect, i.e., the combined effect of cannabis and some other drug or drugs. Furthermore, there were associations between other drugs and reasoning performance which although not significant when controlling the FDR and FWE, nonetheless might become so were a larger sample employed. Indeed, on an unadjusted basis, among the illicit drugs, only cocaine use was significantly associated with non common inferences. Furthermore, as noted above, the very small sample sizes, especially in relation to cocaine severely restricts the power of the analysis to detect a modest correlation of between 0.3 and 0.4 should one actually exist. Thus the implications of the non significant outcomes in Table 2 remain somewhat ambiguous.

An additional limitation that needs to be acknowledged is that in common with much of the existing literature, this study has relied on self-report data in relation to drug use. However, while objective measures would have been desirable, research suggests a high degree of concordance between self-report and objective measures of recent drug use from saliva (Yacoubian \& Wish, 2006) and of longer term use from hair (Scholey et al. 2011; 
Vignali et al. 2012). Furthermore, concordance between self-reports and objective measures of drug use has been demonstrated for multiple illicit drugs (Vignali et al. 2012), cannabis and cocaine (Vignali et al. 2012; Zaldívar, et al. 2009) and ecstasy (Scholey et al. 2011; Yacoubian \& Wish, 2006).

Lastly, it is noteworthy that there is a degree of missing data which is readily apparent comparing the sample sizes associated with the various measures in Table 2 . This was partly due to oversight on our part in that data on long term consumption patterns was misplaced when one of our laboratories relocated. Beyond this, participants were better able to report on the extent of their recent use and make categorical distinctions, e.g., whether or not they had ever used a particular drug, as opposed to confidently reporting longer term trends. In a few instances, responses were missing from the questionnaire possibly due to questions being overlooked. A degree of missing data is not uncommon in studies of this kind (e.g., Bedi \& Redman, 2008; Indlekofer et al. 2009). However, while we wished to avail ourselves of the largest possible sample for each of the comparisons in question, it should be borne in mind that some of the significant associations (or lack of them) reported in Table 2 relate to subsets of the data.

In conclusion, among the illicit drug users in the present study, aspects of recent cannabis use emerged as being associated with poor syllogistic reasoning performance. The effects observed do not appear to reflect simple short term post intoxication effects and the possibility that they may be linked with the concurrent use of tobacco or some other illicit drug cannot be excluded. Aside from the drug related effects, a significant association was observed between IQ (Raven's Progressive Matrices) and syllogistic reasoning performance. 


\section{Disclosures and Acknowledgements}

The authors declare that, except for income received from their primary employers, this research received no specific grant from any funding agency in the public, commercial, or not-for-profit sectors.

All authors have made a significant contribution to the manuscript and have read and approved the final draft.

The authors are not aware of any conflict of interest and do not have any financial interest in this piece of research. 


\section{$\underline{\text { References }}$}

Bedi G Redman J, 2008. Ecstasy use and higher-level cognitive functions: weak effects of ecstasy after control for potential confounds. Psychol Med 38: 1319-1330.

Benjamini Y, Yekutieli D (2001). The control of the false discovery rate in multiple testing under dependency. Ann Stat 29: 1165-1188.

Bland JM, Altman DG (1995). Multiple significance tests: the Bonferroni method. Brit Med J 310: 170 .

Bucciarelli M, Johnson-Laird PN (1999) Strategies in syllogistic reasoning. Cognitive Sci 23: 247-303.

Cunha PJ, Nicastri S, de Andrade AG, Bolla KI. (2010) The Frontal Assessment Battery (FAB) reveals neurocognitive dysfunction in substance-dependent individuals in distinct executive domains: Abstract reasoning, motor programming, and cognitive flexibility. Addict Behav 35: 875-881.

Fernández-Serrano MJ, Pérez-García M, Río-Valle JS, Verdejo-García A. (2010).

Neuropsychological consequences of alcohol and drug abuse on different components of executive functions. J Psychopharmacol 24: 1317-1332.

Fisk JE, Montgomery C, Wareing M, Murphy P. (2005). Reasoning deficits in ecstasy (MDMA) polydrug users. Psychopharmacology 181: 550-559.

Galotti KM. (1989). Approaches to studying formal and everyday reasoning. Psychol Bull 105: 331-351.

Gilhooly KJ, Logie RH, Wetherick NE, Wynn V (1993). Working memory and strategies in syllogistic-reasoning tasks. Mem Cognition 21: 115-124.

Gilhooly KJ, Logie RH, Wynn V (1999). Syllogistic reasoning tasks, working memory, and skill. Eur J Cogn Psychol 11: 473-498. 
Gilinsky AS, Judd BB (1994). Working memory and bias in reasoning across the life-span. Psychol Aging 9: 356-371.

Goel V, Buchel C, Frith C, Dolan RJ (2000). Dissociation of mechanisms underlying syllogistic reasoning. Neuroimage 12: 504-514.

Hadjiefthyvoulou F, Fisk JE, Montgomery C, Bridges N. (2011). Everyday and prospective memory deficits in ecstasy/polydrug users. J Psychopharmacol, 25, 453-464.

Indlekofer F, Piechatzek M, Daamen M, Glasmacher C, Lieb R, Pfister H, Tucha O, Lange KW, Wittchen HU, Schütz CG. (2009). Reduced memory and attention performance in a population-based sample of young adults with a moderate lifetime use of cannabis, ecstasy and alcohol. J Psychopharmacol 23: 495-509.

Jacobsen LK, Pugh KR, Constable RT, Westerveld M, Mencl WE (2007). Functional correlates of verbal memory deficits emerging during nicotine withdrawal in abstinent adolescent cannabis users. Biol Psychiat 61: 31-40.

Jager G, Kahn RS, Van den Brink W, Van Ree JM. Ramsey NF (2006). Long-term effects of frequent cannabis use on working memory and attention: an fMRI study. Psychopharmacology, 185, 358-368.

Jager G, Van Hell HH, De Win MML, Kahn RS, Van den Brink W, Van Ree JM, Ramsey NF (2007). Effects of frequent cannabis use on hippocampal activity during an associative memory task. Eur Neuropsychopharm 17: 289-297.

Johnson-Laird PN (1983). Mental Models. Cambridge, UK: Cambridge University Press. Kanayama G, Rogowska J, Pope HG, Gruber SA, Yurgelun-Todd DA (2004). Spatial working memory in heavy cannabis users: A functional magnetic resonance imaging study. Psychopharmacology, 176(3-4), 239-247. 
Khemlani S, Johnson-Laird PN (2012). Theories of the syllogism: A meta-analysis. Psychol Bull 138: 427-457.

Montgomery C, Fisk JE, Newcombe R, Wareing M, Murphy P. (2005). Syllogistic reasoning performance in MDMA (Ecstasy) users. Exp Clin Psychopharm 13: 137-145.

Morris MW, Nisbett RE (1993). Tools of the Trade: Deductive Schemas taught in psychology and philosophy. In Nisbett, R.E. (Ed), Rules for reasoning, Hillsdale New Jersey: Lawrence Erlbaum. pp228-256

Murphy PN, Wareing M, Fisk JE, Montgomery C (2009). Executive Working Memory Deficits in Abstinent Ecstasy/MDMA Users: A Critical Review. Neuropsychobiology, $60,159-175$.

Nakagawa S (2004). A farewell to Bonferroni: the problems of low statistical power and publication bias. Behav Ecol 15: 1044-1045.

Narum SR (2006). Beyond Bonferroni: Less conservative analyses for conservation genetics. Conserv Genet 7: 783-787

Pike N (2011). Using false discovery rates for multiple comparisons in ecology and evolution. Methods Ecol Evol, 2: 278-282

Politzer G (2011). Solving Natural Syllogisms. In Manktelow, KI, Over, DE, Shira E (Eds), The Science of Reason: A Festschrift for Jonathan St. B.T. Evans. Hove Sussex UK: Psychology Press (pp 17-35)

Raven J, Raven JC, Court JH (1998) Manual for Raven's Progressive Matrices and Vocabulary Scales. Oxford, UK: Oxford Psychologists Press SPM1-SPM95. Reverberi C, Bonatti LL, Frackowiak RSJ, Paulesu E, Cherubini P, Macaluso E (2012). Large scale brain activations predict reasoning profiles. NeuroImage, 59, 1752-1764. 
Reverberi C, Cherubini P, Frackowiak RSJ, Caltagirone C, Paulesu E, Macaluso E. (2010). Conditional and syllogistic deductive tasks dissociate functionally during premise integration. Hum Brain Mapp 31: 1430-1445.

Rodriguez-Moreno D, Hirsch J (2009). The dynamics of deductive reasoning: An fMRI investigation. Neuropsychologia, 47, 949-961.

Scholey AB, Owen L, Gates J, Rodgers J, Buchanan T, Ling J, ... Parrott A (2011). Hair MDMA samples are consistent with reported ecstasy use: findings from a study investigating effects of ecstasy on mood and memory. Neuropsychobiology, 63, 1521.

Shikishima C, Hiraishi K, Yamagata S, Sugimoto Y, Takemura R, Ozaki K, ... Ando J. (2009). Is g an entity? A Japanese twin study using syllogisms and intelligence tests. Intelligence, 37(3), 256-267.

Shikishima C, Yamagata S, Hiraishi K, Sugimoto Y, Murayama K, Ando J. (2011). A simple syllogism-solving test: Empirical findings and implications for g research. Intelligence, 39(2-3), 89-99

Verschueren N, Schaeken W, d'Ydewalle G. (2005). Everyday conditional reasoning: A working memory-dependent trade off between counterexample and likelihood use. Mem Cognition 33: 107-119.

Vignali C, Stramesi C, Vecchio M, Groppi A. (2012). Hair testing and self-report of cocaine use. Forensic Sci Int, 215, 77-80.

Yacoubian GS Jr, Wish ED (2006) Exploring the validity of self-reported ecstasy use among club rave attendees. J Psychoactive Drugs, 38, 31-34.

Zaldívar BF, García MJM, Flores CP, Sánchez SF, López RF, Molina MA. (2009). Validity of the self-report on drug use by university students: correspondence between selfreported use and use detected in urine. Psicothema, 21, 213-219. 
Table 1: Number of Individuals Co-Using Legal and Illegal Drugs

\begin{tabular}{|c|c|c|c|c|c|c|c|c|}
\hline \multirow[t]{2}{*}{ Drug } & \multirow{2}{*}{$\begin{array}{l}\text { Total } \\
\text { number } \\
\text { of Users }\end{array}$} & \multicolumn{7}{|c|}{ Co-Used With: } \\
\hline & & Alcohol & $\begin{array}{l}\text { Amphet- } \\
\text { amine }\end{array}$ & Cannabis & Cocaine & Ecstasy & Ketamine & LSD \\
\hline Alcohol & 101 & - & 19 & 62 & 47 & 52 & 24 & 17 \\
\hline Amphetamine & 20 & 19 & - & 20 & 19 & 19 & 16 & 15 \\
\hline Cannabis & 66 & 62 & 20 & - & 45 & 47 & 24 & 18 \\
\hline Cocaine & 50 & 47 & 19 & 45 & - & 43 & 24 & 18 \\
\hline Ecstasy & 54 & 52 & 19 & 47 & 43 & - & 24 & 17 \\
\hline Ketamine & 25 & 24 & 16 & 24 & 24 & 24 & - & 14 \\
\hline LSD & 18 & 17 & 15 & 18 & 18 & 17 & 14 & - \\
\hline Tobacco & 32 & 31 & 11 & 25 & 20 & 20 & 11 & 8 \\
\hline
\end{tabular}


Table 2: The relationship between syllogistic reasoning and legal and illegal drug use

\begin{tabular}{|c|c|c|c|c|c|}
\hline & \multirow[t]{2}{*}{ Mean } & \multirow[t]{2}{*}{ SD } & \multirow[t]{2}{*}{$\mathrm{n}$} & \multicolumn{2}{|c|}{ Correlation: $r$} \\
\hline & & & & $\begin{array}{l}\text { Common } \\
\text { Inferences }\end{array}$ & $\begin{array}{l}\text { Non Common } \\
\text { Inferences }\end{array}$ \\
\hline \multicolumn{6}{|l|}{ Syllogistic Reasoning } \\
\hline $\begin{array}{l}\text { Common Inferences } \\
(\max 8)\end{array}$ & 4.59 & 2.20 & 105 & & $.375^{* * *}$ \\
\hline $\begin{array}{l}\text { Non Common } \\
\text { Inferences }(\max 6)\end{array}$ & 2.03 & 1.53 & 105 & $.375 * * *$ & \\
\hline \multicolumn{6}{|l|}{ Background Variables } \\
\hline Ravens IQ (max 60) & 45.40 & 8.61 & 104 & $.448 * * *$ & $.250 * *$ \\
\hline Age (years) & 21.33 & 3.14 & 105 & .035 & -.050 \\
\hline \multicolumn{6}{|l|}{ Legal Drugs } \\
\hline \multicolumn{6}{|l|}{ Alcohol } \\
\hline Length of Use (weeks) & 363.10 & 186.02 & 98 & .160 & .021 \\
\hline $\begin{array}{l}\text { Previous } 10 \text { days use } \\
\text { (units) }\end{array}$ & 16.07 & 14.21 & 83 & .035 & .079 \\
\hline Units per week & 13.31 & 11.14 & 100 & .079 & .013 \\
\hline \multicolumn{6}{|l|}{ Tobacco } \\
\hline Length of Use (weeks) & 343.23 & 230.27 & 54 & .126 & -.091 \\
\hline $\begin{array}{l}\text { Previous } 10 \text { days use } \\
\text { (cigarettes) }\end{array}$ & 82.00 & 78.08 & 26 & -.274 & -.292 \\
\hline Cigarettes per day & 8.68 & 7.83 & 34 & $-.405^{*}$ & $-.366^{*}$ \\
\hline \multicolumn{6}{|l|}{ Illegal Drugs } \\
\hline \multicolumn{6}{|l|}{ Total Prior Consumption } \\
\hline Cannabis (joints) & 2427.19 & 4270.51 & 46 & -.151 & .118 \\
\hline Cocaine (lines) & 716.91 & 1234.12 & 26 & .116 & .198 \\
\hline Ecstasy (tablets) & 675.30 & 1345.78 & 41 & -.137 & -.038 \\
\hline \multicolumn{6}{|l|}{ Long Term Average } \\
\hline \multicolumn{6}{|l|}{ Dose Per Session } \\
\hline Cannabis (joints) & 2.71 & 2.05 & 46 & $-.324 *$ & -.101 \\
\hline Cocaine (lines) & 5.73 & 4.34 & 26 & .019 & .032 \\
\hline Ecstasy (tablets) & 3.82 & 5.57 & 41 & $-.351 *$ & -.237 \\
\hline \multicolumn{6}{|l|}{ Long Term Average } \\
\hline \multicolumn{6}{|l|}{$\begin{array}{l}\text { Frequency (times per } \\
\text { week) }\end{array}$} \\
\hline Cannabis & 2.19 & 2.35 & 46 & $-.355^{*}$ & .001 \\
\hline Cocaine & 0.71 & 0.63 & 26 & -.314 & -.167 \\
\hline Ecstasy & 0.72 & 1.15 & 41 & -.185 & .038 \\
\hline \multicolumn{6}{|l|}{ Amount Consumed in } \\
\hline \multicolumn{6}{|l|}{ Previous 30 Days } \\
\hline Cannabis (joints) & 11.75 & 34.65 & 62 & $-.389 * * *$ & -.157 \\
\hline Cocaine (lines) & 8.24 & 12.99 & 38 & -.148 & $-.290 \dagger$ \\
\hline Ecstasy (tablets) & 4.30 & 10.80 & 54 & -.206 & -.177 \\
\hline \multicolumn{6}{|l|}{$\begin{array}{l}\text { Current Frequency of } \\
\text { Use (times per week) }\end{array}$} \\
\hline Cannabis & 0.80 & 1.59 & 62 & $-.328 * *$ & -.132 \\
\hline Cocaine & 0.40 & 0.47 & 37 & $-.281 \dagger$ & $-.363^{*}$ \\
\hline Ecstasy & 0.28 & 0.44 & 54 & -.040 & -.112 \\
\hline
\end{tabular}


Figure 1 : Plot of Correct Common Syllogistic Responses Against Recent Consumption of Cannabis

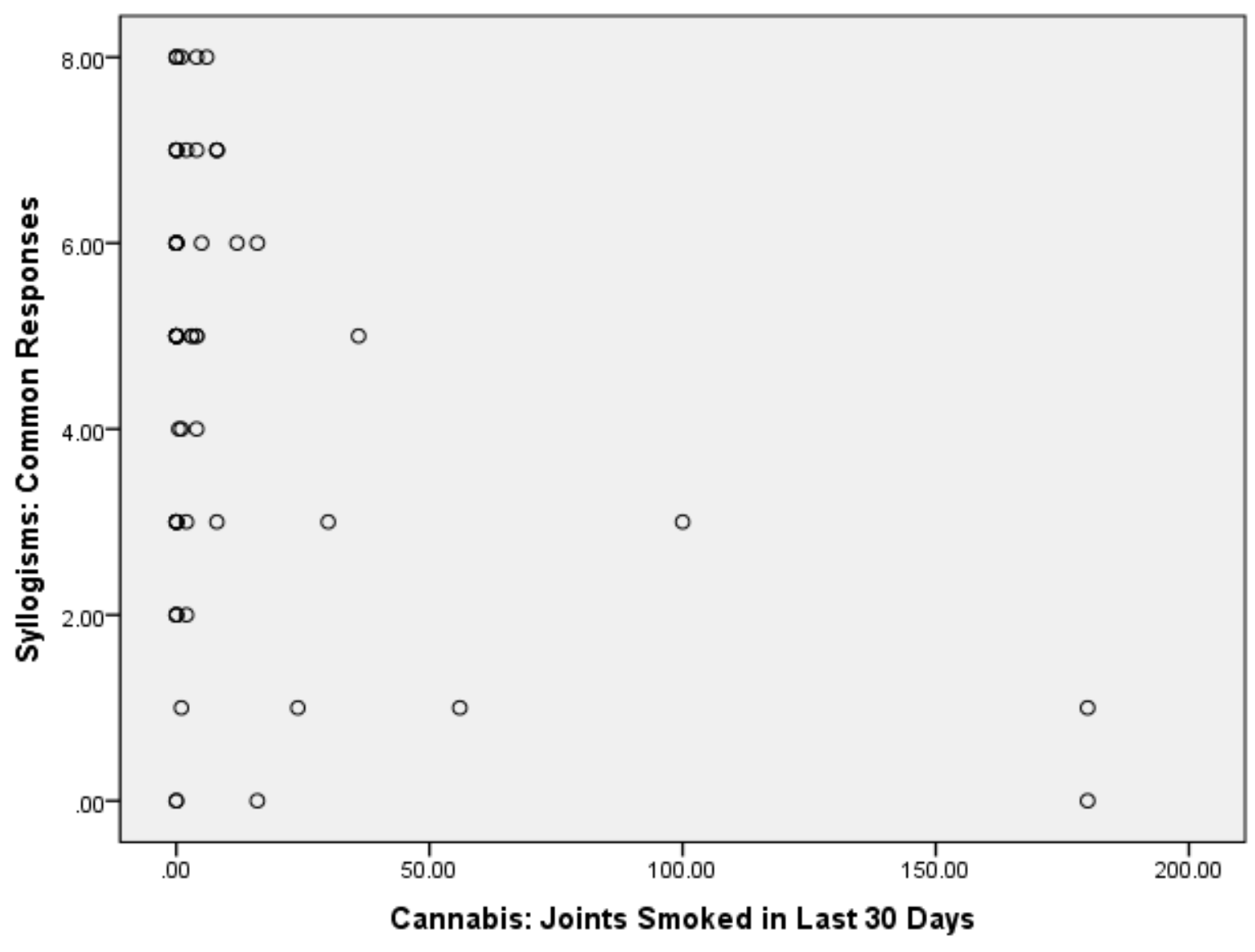


Figure 2 : Plot of Correct Common Syllogistic Responses Against Recent Consumption of Ecstasy

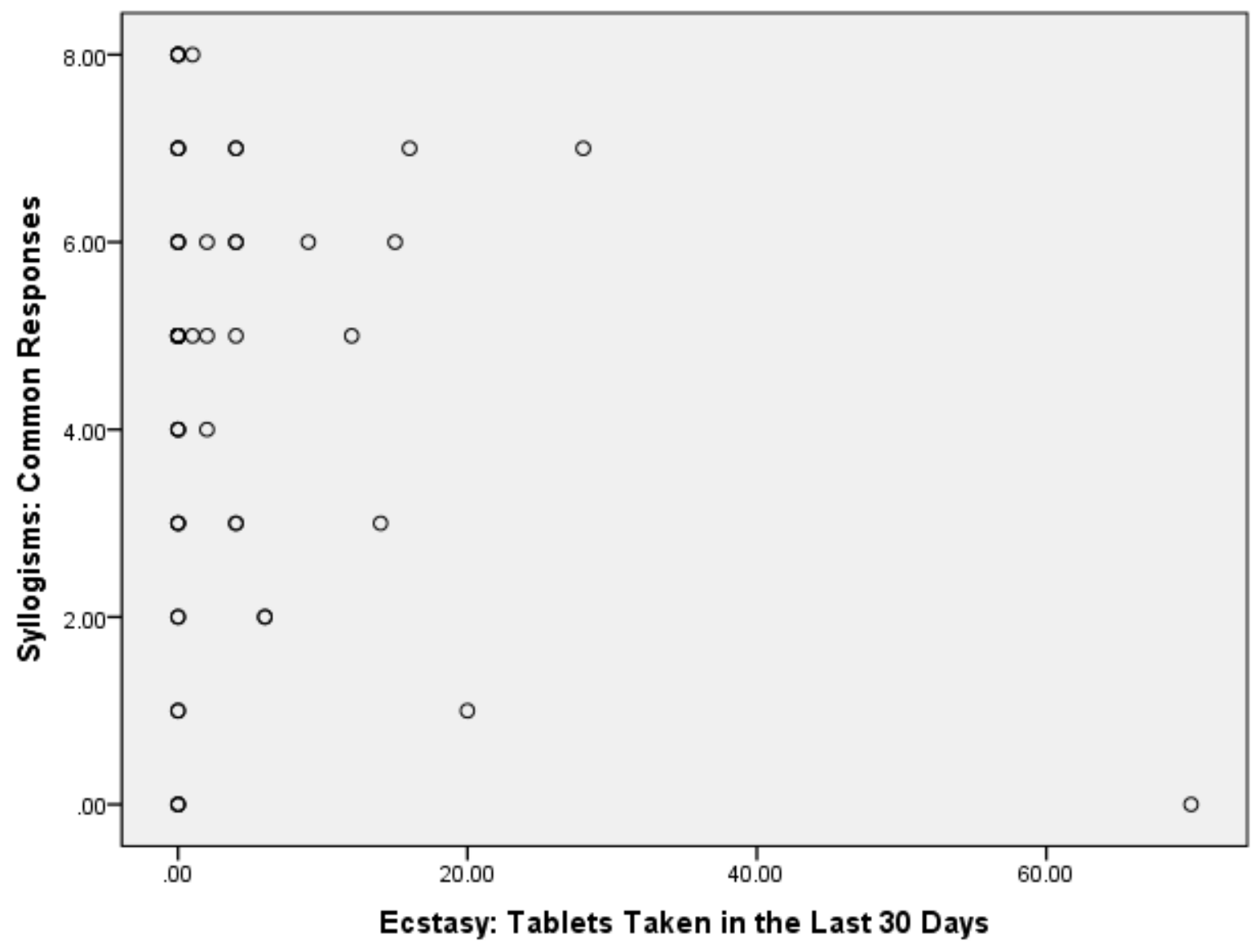


Figure 3 : Plot of Correct Common Syllogistic Responses Against Recent Consumption of Cocaine

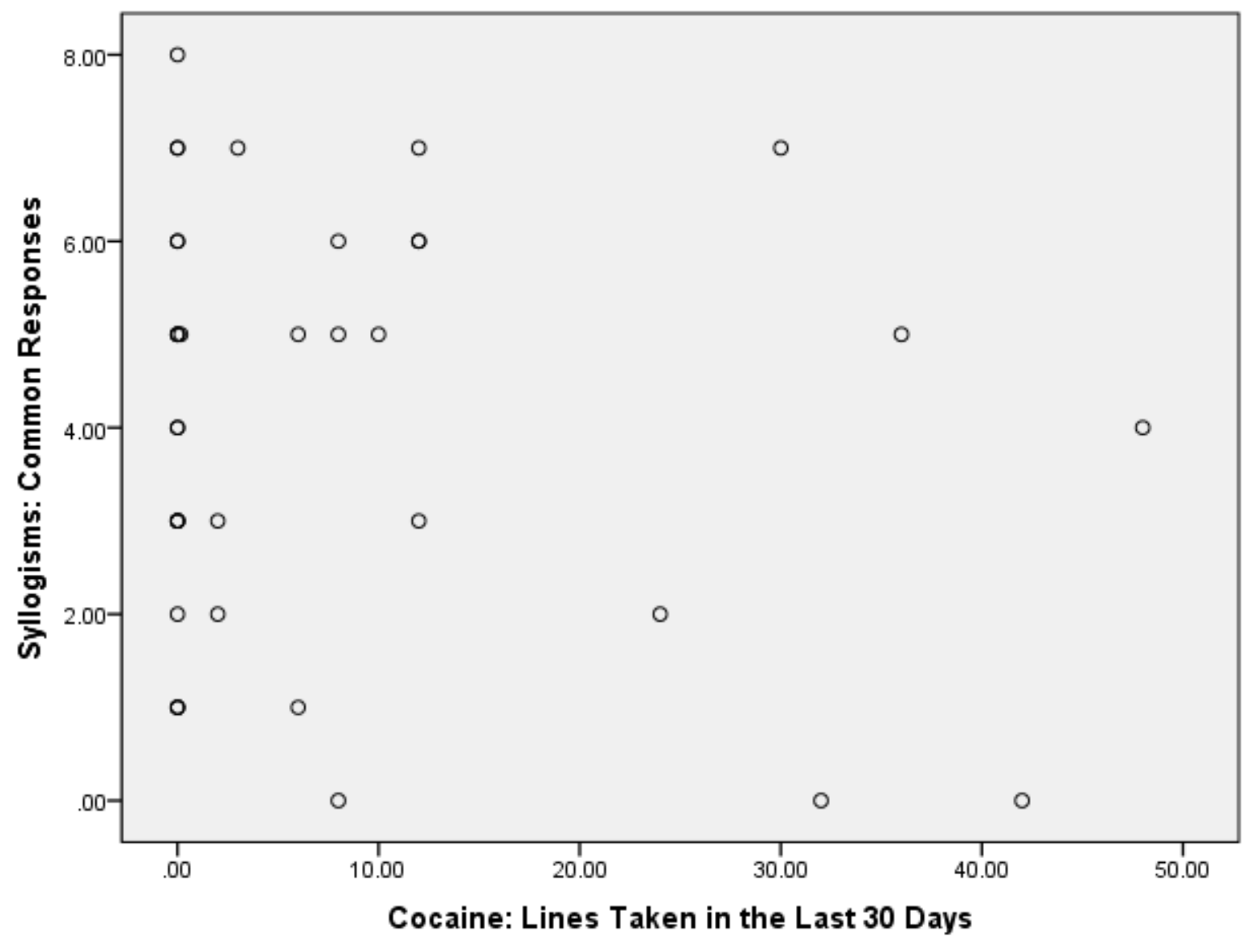


Figure 4 : Plot of Correct Common Syllogistic Responses Against Current Frequency of Cannabis Use

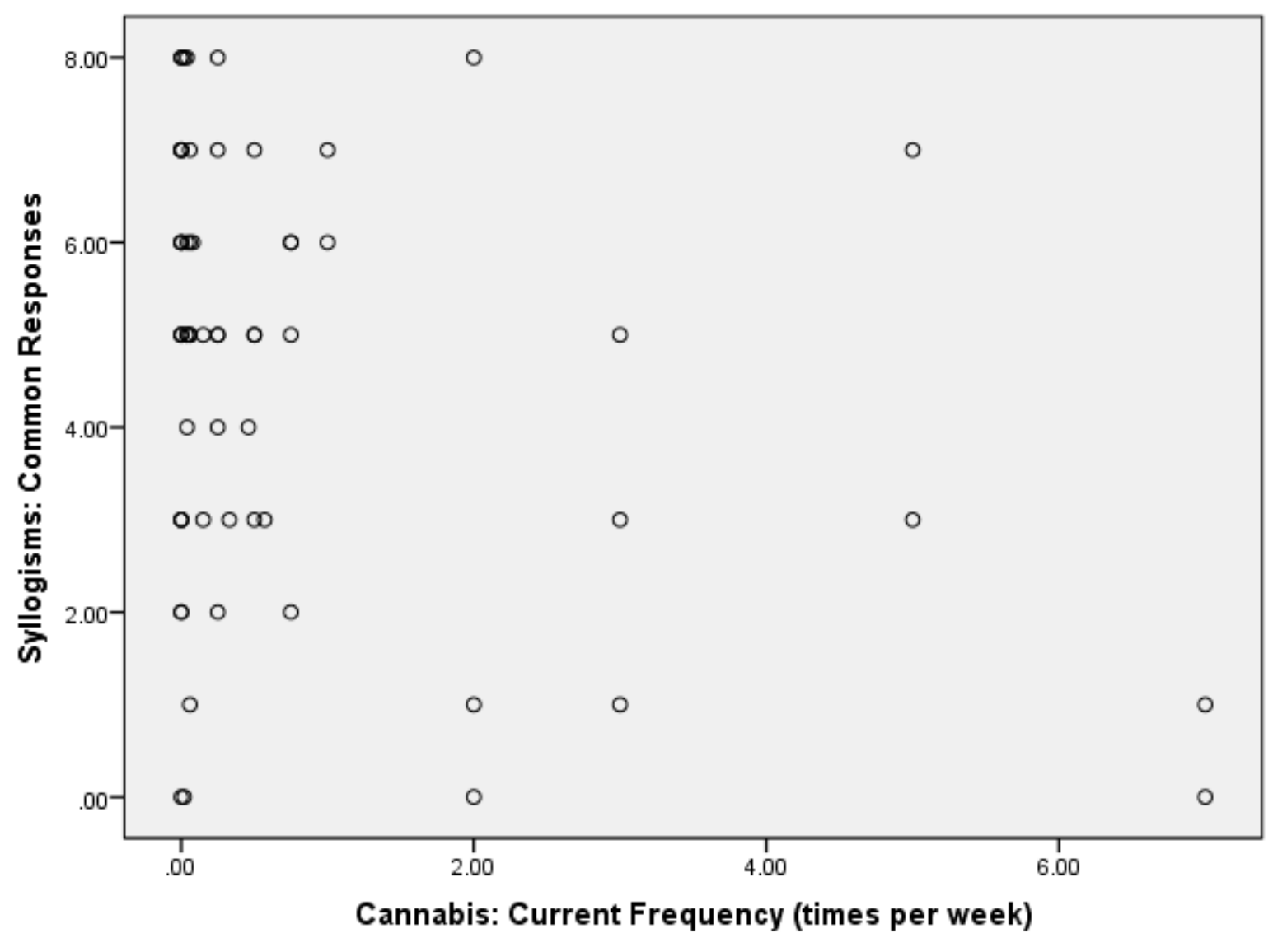


Figure 5 : Plot of Correct Common Syllogistic Responses Against Current Frequency of Ecstasy Use

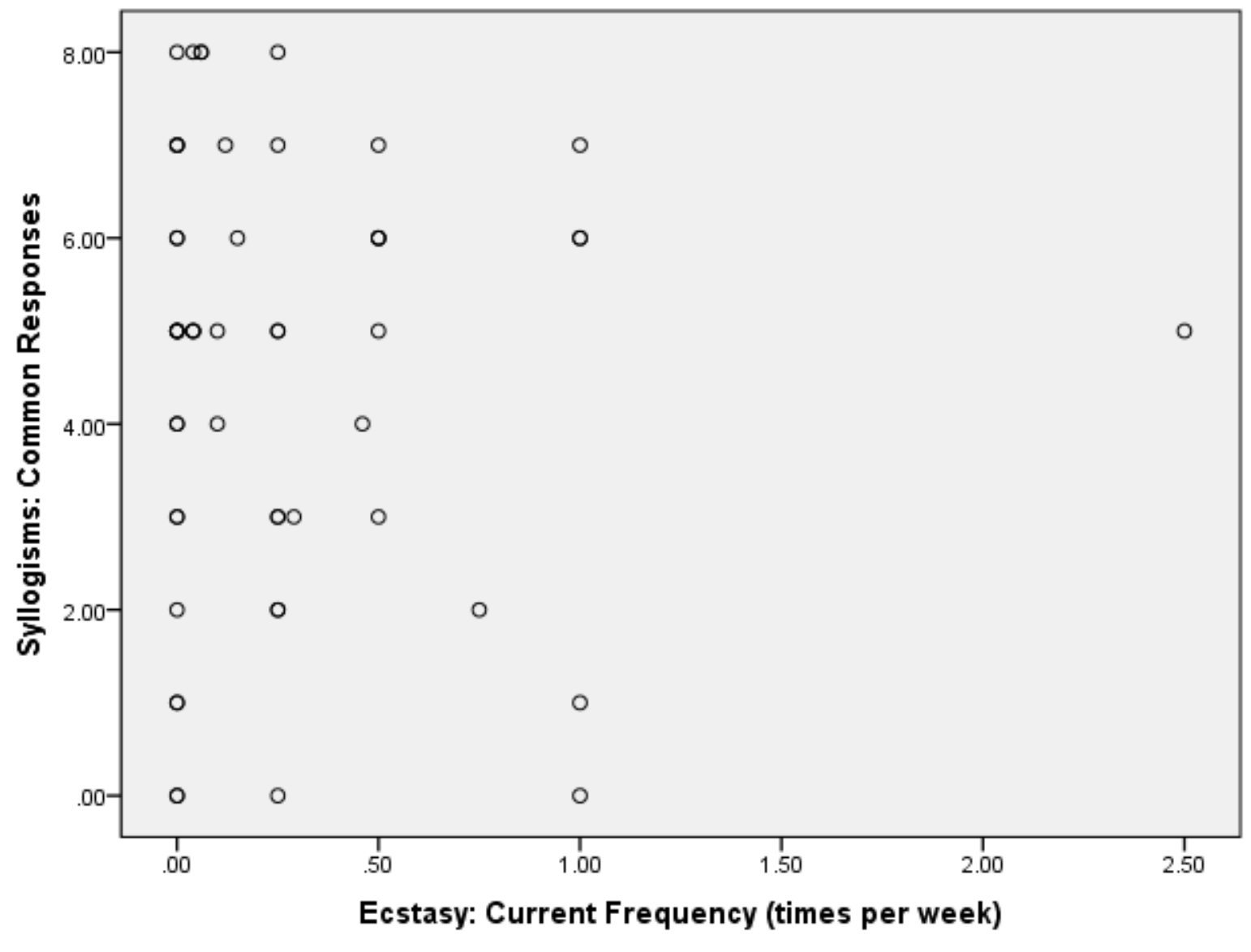


Figure 6 : Plot of Correct Common Syllogistic Responses Against Current Frequency of Cocaine Use

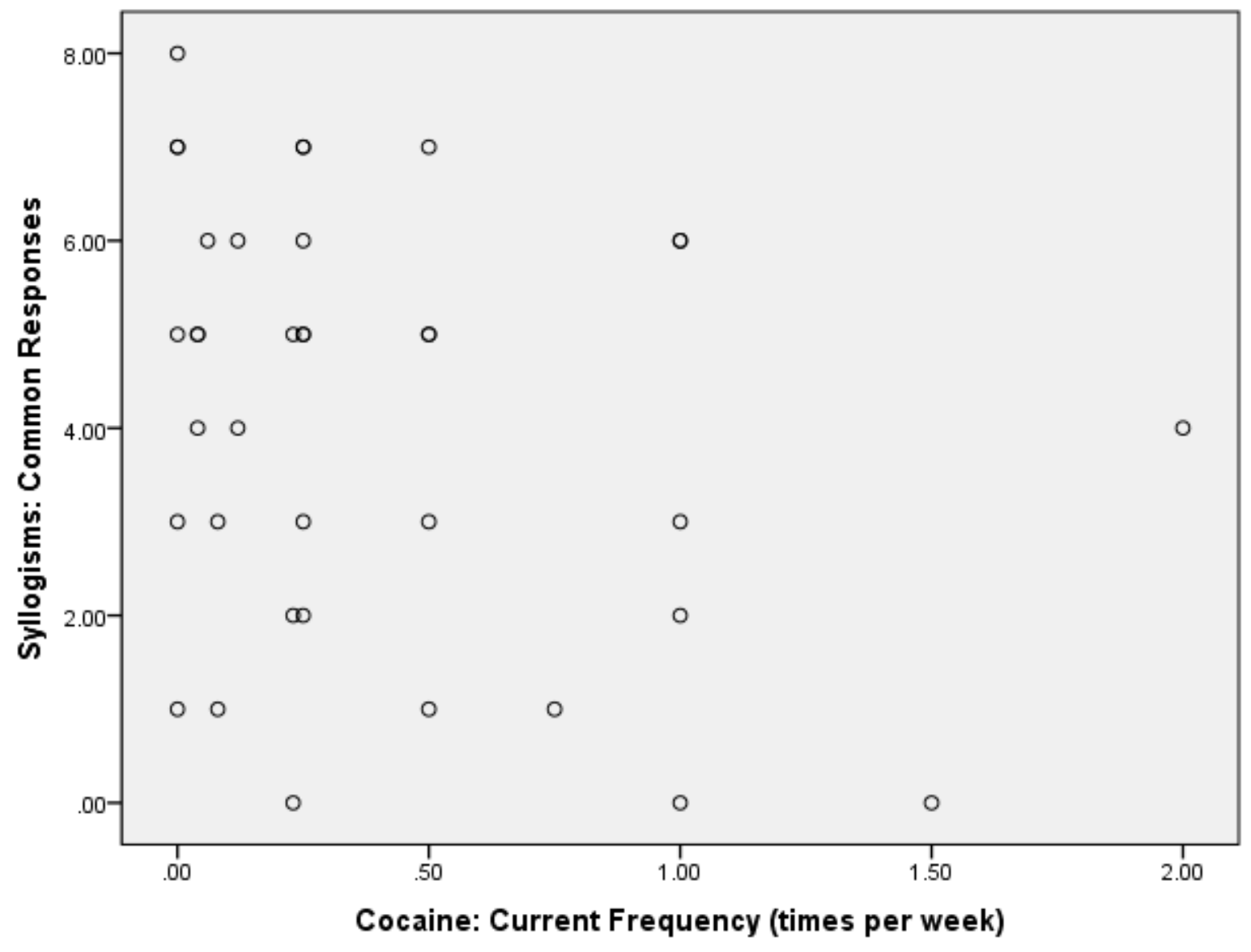


Some of the B are A

All of the B are C

All of the A are B

None of the $\mathrm{B}$ are $\mathrm{C}$

Some of the B are A

All of the $C$ are $B$

All of the B are A

Some of the $\mathrm{C}$ are $\mathrm{B}$

Some of the A are B

All of the B are C

All of the B are A

All of the $C$ are $B$

All of the A are B

None of the $\mathrm{C}$ are $\mathrm{B}$

None of the A are B

All of the $\mathrm{C}$ are $\mathrm{B}$

All of the A are B

All of the $B$ are $C$ 